\title{
Chelating Effect of Cellulose Acetate Hydrogel Crosslinked with EDTA Dianhydride Used as a Platform for Cell Growth
}

\author{
Anna Melero, ${ }^{1}$ Andre Senna, ${ }^{2}$ Juliana Domingues $\left(D,{ }^{3}\right.$ Adriana Motta $\left(D,{ }^{3}\right.$ Moema Haussen, ${ }^{3}$ \\ Antonio Riul Junior, ${ }^{3}$ Eliana Duek, ${ }^{3}$ and Vagner Botaro ${ }^{1,2}$ \\ ${ }^{1}$ Federal University of Ouro Preto-REDEMAT, Ouro Preto, Brazil \\ ${ }^{2}$ Federal University Sorocaba, UFSCAR, São Carlos, Brazil \\ ${ }^{3}$ Biomaterials Laboratory, PUCSP, São Paulo, Brazil
}

Correspondence should be addressed to Juliana Domingues; almeidajad_bio@hotmail.com

Received 28 December 2018; Revised 25 April 2019; Accepted 14 May 2019; Published 15 September 2019

Academic Editor: Michelina Catauro

Copyright (C) 2019 Anna Melero et al. This is an open access article distributed under the Creative Commons Attribution License, which permits unrestricted use, distribution, and reproduction in any medium, provided the original work is properly cited.

The use of biomaterials capable of achieving controlled release of drugs or chemical and physical interactions with living organisms causes these new materials to be studied in depth to better target their future applications. An example of this is the use of materials that have in their physical structure elements with chelating effects, capable of interacting with the ions and cations present in the cell culture media, drugs, and other elements capable of interacting with the human body. In this work, cellulose acetate hydrogel (HAC) crosslinked with EDTAD (ethylenediaminetetraacetic acid dianhydride) showed a significant chelating effect capable of altering the adhesion of mesenchymal cells in the first days of in vitro tests. This result became our main question in this work, and by using new cell viability assays, it was verified that the hydrogel interacted with the culture medium, removed the salts present, such as $\mathrm{Ca}^{2+}$ and $\mathrm{Mg}^{2+}$, and promoted a decrease in the amount of cells adhered to the material but not cell death. After saturation of $\mathrm{Ca}^{2+}$ and $\mathrm{Mg}^{2+}$, we obtained an improvement in cell adhesion. Electrochemical impedance spectroscopy (EIS) results also verified the dynamics of linked or mobile loads in the volume and interface regions of HAC-EDTA and the presence of characteristic bands related to the interaction between calcium and magnesium present in the culture medium with HAC-EDTA. The DMA traction test showed that there was no dynamic-mechanical change in the structure of the material at temperatures between 20 and $60^{\circ} \mathrm{C}$, which is ideal for this studied biomaterial. The purpose of these tests is to aid in the characterization of future biomaterials, where the physicochemical interaction of some of them is often confused with a false cytotoxicity, which in fact may only be an easily identifiable and adaptable structural feature.

\section{Introduction}

The multidisciplinary collaboration enables the development of new materials that can improve, for example, regenerative processes in tissue engineering, the production of new medical devices, and also the expansion and application of synthetic materials for the production of biological products for the treatment of patients [1].

These practices contributed to new ideas and bolder methods, where not only objects could be developed and replaced but also man could benefit physically from these devices, since in a discrete but not less important way science, technology, and the application of materials are present in the history of the world, such as embalming techniques, postwar surgical techniques, and even dressings and contact lenses that are currently used [2], and currently, we can monitor research related to hydrogels for cartilage regeneration [3].

Hydrogels are polymers formed by three-dimensional interconnected networks capable of absorbing and retaining large amounts of water, thus promoting good biocompatibility and biodegradability [4]. To expand the limitations of the use of bone tissue autograft, a strategy would be to use biomaterials capable of increasing the capacity for bone regeneration.

Thus, the use of drug-bearing hydrogels for bone and cartilage regeneration has been studied in order to find a biomaterial model that is ideal for this type of application 
$[3,5]$. In addition, hydrogels have been further studied because they minimize invasive procedures and are inserted in regions located directly at the site of interest [6].

Gelli et al. [5] in their recent study present results related to amorphous calcium and magnesium phosphates that recently attracted great attention in view of their biological relevance. In other medical aspects, the importance of understanding of this process opens up to a series of intriguing aspects also from a physicochemical perspective.

Among the promising hydrogels in the field of biomaterials, mainly for applications in tissue engineering $[7,8]$, a new class of cellulose acetate-based hydrogels crosslinked with sequestering agents has been extensively studied for the controlled release of drugs and interfacial bonding of tissue implants $[9,10]$.

In three-dimensional networks, this increase of covalent bonds forms normally linear subsets, which are responsible for separating the adjacent junction points [11]. By means of junction points, the macromolecules interact with each other due to the covalent bonds [11].

The formation of polymer networks is determined by the type of synthesis performed and by the presence of reagents capable of branching the molecular structures, thus forming the polymer networks, which can be formed by crosslinking or functional group polymerization (endlinking) $[11,12]$.

Ethylenediaminetetraacetic acid (EDTA) is an aminopolycarboxylic acid and a water-soluble solid, which in processes and synthesis forms a complex precursor for a low-temperature synthesis of cathodic materials.

The chelating agents can be defined as chemical compounds that chelate metal ions, such as calcium, magnesium, and iron, and can form complex compounds [13], and the main reason for using EDTA as a chelating agent is its ability to chelate the multivalent cations, which represents the stability in the binding strength between the chelate and the metal ion (cation), i.e., the higher the value is, the more stable the compound is, and the high stability means high affinity of the chelating agent to this cation [13].

It is possible to find in the literature studies involving EDTA as a chelating agent, such as the work of Mahmoud et al. [13], where an improved EDTA/seawater system for oil recovery was introduced in sandstone tanks. Improved oil recovery fluid utilizing EDTA/seawater presents high $\mathrm{pH}$ low-level chelating agents when prepared in seawater, which present in intermediate layers at the rock/brine and brine/oil interfaces without affecting the integrity of the rock and dissolving the carbonated minerals [14].

In addition, it is possible to observe the stability of the EDTA in high temperature and high $\mathrm{pH}$. Mahmoud [14] showed that EDTA has the ability to restrict the reactivity of different cations, especially $\mathrm{Ca}^{2+}$ and $\mathrm{Fe}^{2+}$, which is proved to be the most important feature of EDTA to increase oil recovery.

Synthetic polymers with hydrophilic properties that contain water in their structure are considered ideal for these types of studies, such as hydrogels $[15,16]$, which are often similar to the extracellular matrix molecules (also produced by cells) and have been the focus of many studies.

Hydrogels have a flexible consistency, and they are nonabrasive, permeable, and, in some cases, biocompatible.
These crosslinked materials are capable of absorbing or exchanging fluids with living organisms $[17,18]$, and these characteristics have become extremely attractive to be applied as biomaterials. Their structure resembles the components of the organism and, in an aqueous medium, protects growing cells, regulates cell function, and helps in the diffusion of nutrients and growth factors [19].

Some applications of hydrogels in biomedical fields are contact lenses, scaffolds as a complement to tissue engineering, hydrophilic membranes for controlled drug release $[3,16,20]$, cartilage implants and dressings for the treatment of burns, and conductive polymers in bioelectronics [21].

Polymer materials used as a platform for cell growth in general should have a surface suitable for cell adhesion and should not interact with the culture medium [22], and for that, during the cell culture assay, an important factor of the process is the culture medium, which is composed of salts enriched with amino acids and other components essential for cell growth.

Among the minerals that make up the culture medium, some play essential roles in the body; for example, calcium and magnesium $[23,24]$ play essential roles in the regulation of immune function [25].

Calcium is an important factor involved in the innate immune system, which is responsible for inducing the oxidative explosion or the respiratory explosion process, in which the superoxide anion $\left(\mathrm{O}_{2-}\right)$ is produced to digest enveloped pathogens [25]. However, magnesium acts as a cofactor in the synthesis of immunoglobulins, C3 convertase (complement system), adhesion of immune cells, antibodydependent catalysis, T-helper adherence, and B cells [26, 27] and has an excellent osteogenic activity [28], and there are studies that have demonstrated antitumor activity of magnesium among other minerals associated with calcium [29].

Absence of these salts in the culture medium can affect cell adhesion and cell division and impair the progress of the experiment. One of the possibilities of deficiency of this free calcium and magnesium in the culture medium can be attributed to materials whose structure is crosslinked with other materials, with a chelating effect, such as the hydrogel used in the development of HAC-EDTA [12], which has EDTA.

The hydrogels that present metric networks with the capacity to retain water inside the porous structure [12] show possibilities in biomedical applications; for example, hydrogels based on natural polymers, including cellulose, are of particular interest due to their hydrophilicity, biocompatibility, and biodegradability [30].

HAC-EDTA is a new polymeric material developed in our research group [12]. It presents features of high degree of swelling, amphoteric character, and low production value, has more attractive physical, chemical, and thermodynamic properties, and has possible application as a biomaterial, which must be evidenced in this work as something unheard of, since this material was never used as a biomaterial until now.

Its amphoteric character draws attention to applications related to controlled release of drugs in several types of treatments. There are currently no reports in the literature 
on cellulose acetate hydrogels crosslinked with EDTA dianhydride whose possible chelating effect has been studied for applications such as biomaterials that have the ability to remove salts from the culture medium and release them later.

Knowing that HAC-EDTA exhibits some of the characteristics mentioned above, it was observed that during the cell culture assays, there was a decrease in the amount of cells and that there was no cell death since many cells were present in the polystyrene wells and some cells were not adhered. It was therefore questioned whether the material was cytotoxic or whether the chelating effect could be interacting with the salts present in the culture medium, thus causing change in results and even a false result of cytotoxicity.

Preliminary studies of the group left some doubts regarding the interaction of calcium and magnesium with HAC-EDTA. For this reason, more tests were carried out on these hypotheses. The possibility of adsorption of $\mathrm{Ca}^{+2}$ and $\mathrm{Mg}^{+2}$ by EDTA, widely known as a chelator, that are present in the structure of the material, allows controlled release of these nutrients during the final stage of cell culture, which implies an increase in the amount of $\mathrm{Ca}^{2+}$ and $\mathrm{Mg}^{2+}$ present in the HAC-EDTA structure due to the chelating effect of EDTA, thus promoting better cell adhesion on the surface of the hydrogel. Some characterization studies were also performed to support the results of this work. It is fundamental to understand this dynamics of interaction because this material has a main function to be used as a biomaterial, and all possibilities of interaction must be very well characterized so that it is not confused with false results of toxicity of the material.

\section{Materials and Methods}

2.1. HAC-EDTA Synthesis. The HAC-EDTA in question is a polymer having crosslinked chains, and its polymer chains are linked together by chain segments joined by covalent primary forces developed by Senna et al. [12].

The material is composed of cellulose acetate with the degree of substitution (GS) 2 and 5 and EDTAD, with cellulose acetate being the main base of the hydrogel. The material is synthesized by crosslinking cellulose acetate chains with EDTAD acting as a crosslinking agent both in the medium of dimethylformamide as solvent, and after homogenization, is added triethylamine as the catalyst. For the production of thin films of HAC-EDTA, casting process is carried out using glass plates and curing process is performed for 10 days (Figure 1).

2.2. Preparation of HAC-EDTA Samples. For the molding of the materials and preparation of the test specimens after the synthesis and gelation reaction of the HAC-EDTA, Petri dishes were used to prepare membranes, as they allow thicker materials, and glass plates with dimensions of $50 \mathrm{~cm} \times 50 \mathrm{~cm} \times 0.4 \mathrm{~cm}$ were used for the preparation of films; after the curing process of the hydrogel, which is already crosslinked, the material is milled to obtain particles. The test specimens for cell viability tests (film) and metal adsorption tests (membrane) were cut into $0.5 \mathrm{~cm}$ discs (Figure 2).

2.3. Fourier-Transform Infrared (FTIR) Spectroscopy. FTIR analysis is an ideal technique for quantitative analysis of chemical processes in real time. With the use of a remote probe and spectrometers, the FTIR allows to perform analysis of functional groups.

The material used for analysis was the HAC-EDTA film after the cell culture assay to verify the presence of proteins and drugs retained in the hydrogel structure and thus to evaluate the possible changes in the bands of the material after loading. The analysis was performed at the Campus Sorocaba Laboratory of PUC-SP, using the PerkinElmer Spectrum 400 FT-IR 400 FT Mid-IR Spectrometer with frequencies between 400 and $4000 \mathrm{~cm}^{-1}$. Five dry samples (film) were analyzed by the ATR method.

2.4. Impedance Spectroscopy. Impedance spectroscopy is an interesting technique to characterize the electrical behavior of solid materials, such as those developed in materials engineering. The impedance tests were performed at the "Gleb Wataghin" Institute of Physics at Unicamp, under the supervision of Prof. Dr. Antonio Riul Júnior. The technique used in this measurement test consisted of placing the HACEDTA sample under evaluation between two electrodes, applying an electrical stimulus, and observing the results.

An alternating voltage of the sinusoidal type was used, and the real and imaginary parts of the complex impedance were measured as a function of frequency.

The impedance spectroscopy measurements were performed on a Solartron impedance analyzer (model 1260), in a frequency range of $1 \mathrm{~Hz}$ to $1 \mathrm{MHz}$, with a fixed voltage of $20 \mathrm{mV}$. The purpose of this test was to verify the variation of the electric capacitance as a function of frequency. In impedance spectroscopy, the measurements are performed by varying the fixed frequency of the applied signal on the interdigitated electrodes.

For the analysis of the results, graphs of the real part and the imaginary part of the impedance as a function of frequency were computed, composing the impedance spectrum for that device formed with the sample of the hydrogel and the two electrodes.

2.5. Dynamic Mechanical Analysis (DMA). Dynamic mechanical tensile analyses were performed on HAC-EDTA samples in the film form, with dimensions of $9.0400 \times 7.6900 \times$ $0.5800 \mathrm{~mm}$, and subjected to sinusoidal deformation with an amplitude of $240 \mu \mathrm{m}$ at a constant frequency of $1 \mathrm{~Hz}$, at a temperature in the range of $-50^{\circ} \mathrm{C}$ to $250^{\circ} \mathrm{C}$, and at a heating rate of $2^{\circ} \mathrm{C} / \mathrm{min}^{-1}$, using a tensile system in accordance with ASTM D 4065-2001.

The samples were analyzed at the Federal University of São Carlos, Campus Sorocaba, using dynamic mechanical analyzer (DMA) equipment from TA Instruments, model Q800, and tensioning and cooling claw was performed using a chiller from TA Instruments. 


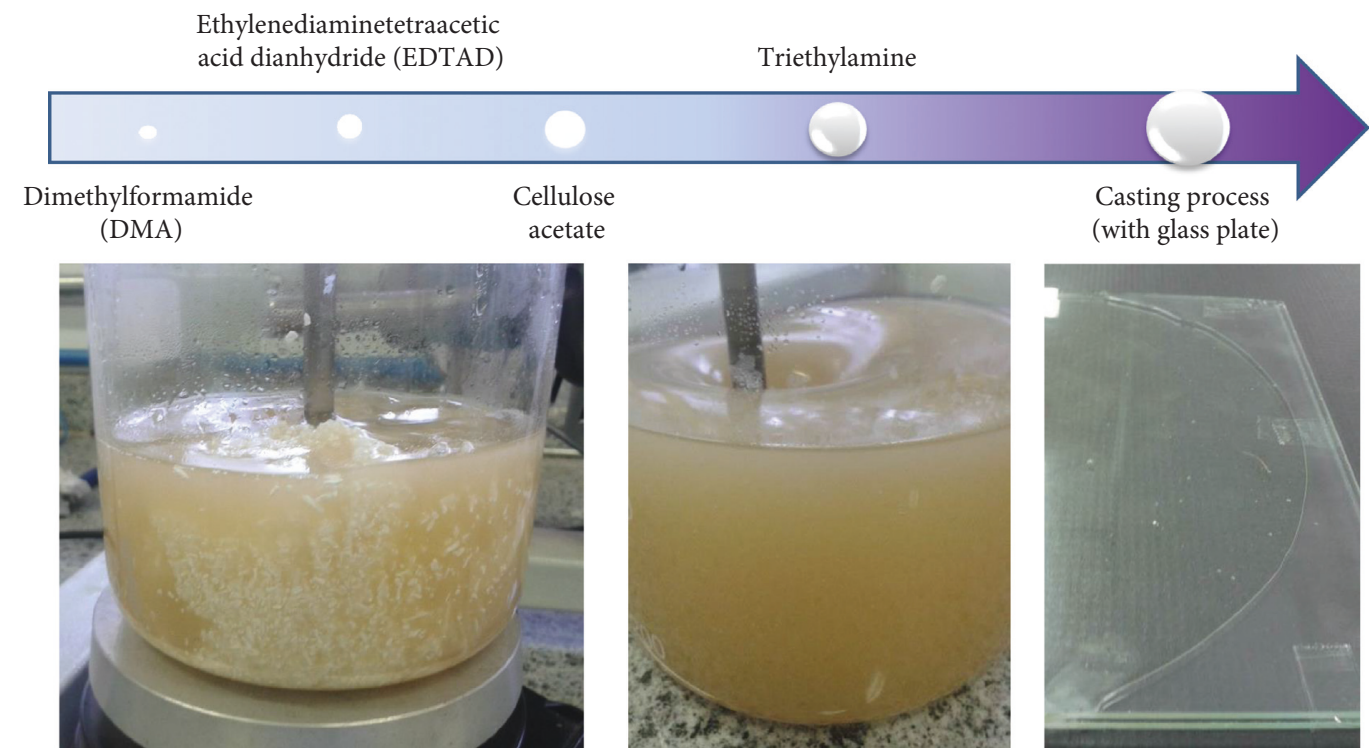

FIgURE 1: Synthesis of HAC-EDTA. Cellulose acetate and EDTAD are dissolved in DMF, then the catalyst is added, and the synthesis is finalized by the casting process.

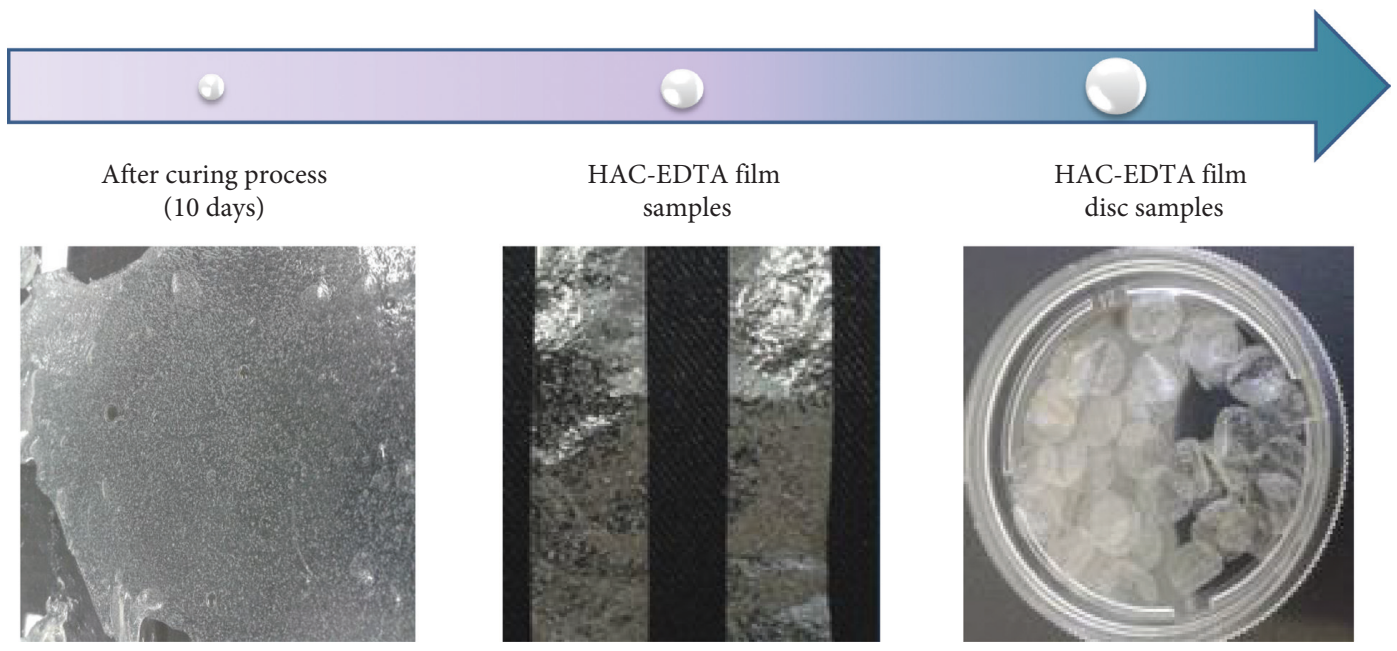

FIgURe 2: Preparation of HAC-EDTA (film and disc) samples after the curing process.

2.6. Cell Viability Assay with HAC-EDTA Saturated with $\mathrm{Ca}^{2+}$ and $\mathrm{Mg}^{2+}$. Adipose-derived mesenchymal cells (ASC, ATCC ${ }^{\circledR}$ PCS-500-011) were cultured to evaluate the cell viability by direct contact in the polymeric device under study (HAC-EDTA). After being cultured routinely, cells were grown in Dulbecco's modified Eagle's medium (DMEM), supplemented with 10\% fetal bovine serum (FBS) containing $1 \%$ antibiotics (penicillin-streptomycin) and $4 \mathrm{mM}$ L-glutamine. The cell lines were incubated at $37^{\circ} \mathrm{C}$ in $5 \% \mathrm{CO}_{2}$ and transferred in new culture bottles with trypsinEDTA when co-confluency was reached. After sterilization, saturated HAC-EDTA samples of $\mathrm{Ca}^{+2}$ and $\mathrm{Mg}^{+2}$ were maintained in a $37^{\circ} \mathrm{C}$ incubator at $5 \% \mathrm{CO}_{2}$ under ambient condition for $1 \mathrm{~h}$ in $200 \mu \mathrm{l}$ of DMEM prior to each experiment. Subsequently, the DMEM medium was withdrawn, and a cell suspension at the concentration of $1 \times 10^{4}$ cells $/ \mathrm{ml}$ was added to each well.
The well plates were incubated at $37^{\circ} \mathrm{C}$ in $5 \% \mathrm{CO}_{2}$. The hydrogel saturated with $\mathrm{Ca}^{2+}$ and $\mathrm{Mg}^{2+}$ previously acclimated to the DMEM was used as a platform to the adhered cells. Wells without samples were used as negative control (CTRL$\mathrm{N}$ ), and their results were considered as $100 \%$ cell viability. As a positive control (CTRL-P), HAC-EDTA membranes without saturation of $\mathrm{Ca}^{2+}$ and $\mathrm{Mg}^{2+}$ were used. The assays were divided into triplicates after total saturation of $\mathrm{Ca}^{2+}$ and $\mathrm{Mg}^{2+}$ in HAC-EDTA. A triplicate of the negative control and the positive control were also performed. After the respective incubation times $(1,7$, and 14 days), the medium was removed and the wells were washed with DMEM.

2.7. Confocal Laser Scanning Microscopy. For confocal laser microscopy observations, cells adhered in samples were fixed with $4 \%$ formaldehyde and permeabilized with triton 
X-100, and after treatment with PBS, cells were immersed and labeled in DAPI containing Fluoroshield antifade (Sigma-Aldrich), and directly labeled by phalloidin conjugated with Alexa Fluor 647 (Invitrogen, Thermo Fisher, USA) to identify actin cytoskeleton. The samples were observed by the photomultiplier tube mode using laser lines $405 \mathrm{~nm}$ and $638 \mathrm{~nm}$ to detect signals of DAPI (blue) and Alexa Fluor 647 (red), respectively. Fluorescent excitation and emission spectra were set according to the instruction of the manufacturer of each fluorophore. The acquisition mode was set to generate $512 \times 512$ pixel images, and pinhole aperture was set to $53 \mu \mathrm{m}$ to obtain optical sections of $2 \mu \mathrm{m}$ when under 20x objective observations. Images were acquired by 10 or $100 \mathrm{~Hz}$ scan speed for $2 \mathrm{D}$ or $3 \mathrm{D}$ images, respectively, and their axial resolution varied from 0.3$0.5 \mu \mathrm{m}$. The Z-stack mode performed the reconstructed slices up to $50 \mu \mathrm{m}$ depth in the xyz scanning mode.

\section{Results and Discussion}

3.1. Impedance Spectroscopy. One of the characteristics of HAC-EDTA is its amphoteric character presented by Senna et al. [12]. This characteristic provides room for, where it still allows, new analyses, such as checking the electrical behavior of the material.

Electrochemical impedance spectroscopy (EIS) was used in this work to investigate the dynamics of bound or mobile charges in the volume and interface regions of HAC-EDTA.

Therefore, it is possible to observe in Figure 3 that the behavior of the impedance module up to approximately $50,000 \mathrm{~Hz}$ is predominantly capacitive and can be observed by the presence of a decreasing line in the graph. In this case, the system can store electrical energy, characterizing the hydrogel's ability to store loads in this frequency range.

At higher than $50,000 \mathrm{~Hz}$, it can be observed in the impedance module as a line parallel to the $x$-axis (Figure 4) the behavior is resistive, indicating that, in this frequency range, we have the system behaving more like a resistor; that is, it opposes handling of cargo carriers.

Electrochemical impedance spectroscopy (EIS) is an important technique for the characterization of a wide variety of electrochemical systems, as in the case of the bonds formed by the chelating effect of EDTA present in the structure of HAC-EDTA and for the determination of the contribution of individual electrode or electrolyte processes in these systems.

The electrochemical impedance spectroscopy technique is an option for biomaterial characterization, such as studies related to implant coatings [31], using calcium phosphate synthesized with magnesium alloys, in which the reduction of the corrosion effect in contact with fluids of the human body should be investigated.

Impedance spectroscopy can be considered a useful tool for the characterization of the intrinsic electrical properties of a biomaterial or its interface. Because of the possibility of interaction between the connected loads or moving crosslinking regions present in HAC-EDTA, the best methodology to verify these results was the use of impedance

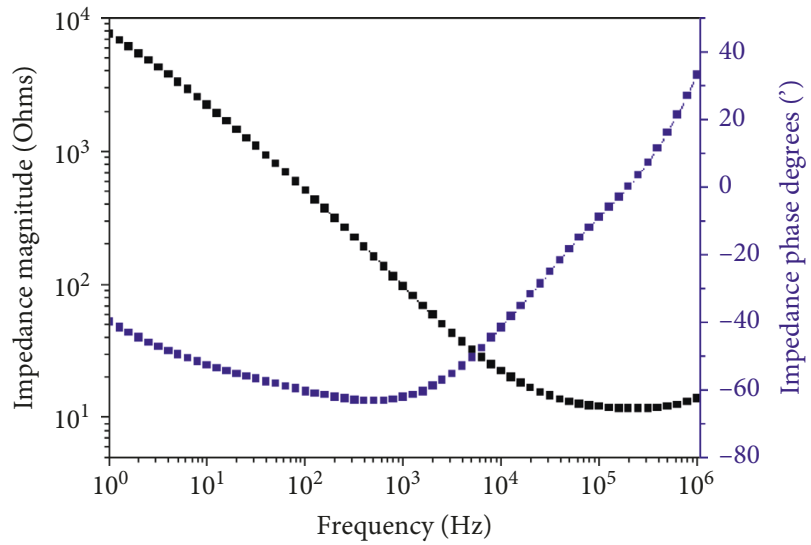

Figure 3: HAC-EDTA impedance spectrum, whose line decay exhibits storage capacity of up to approximately $50,000 \mathrm{~Hz}$.

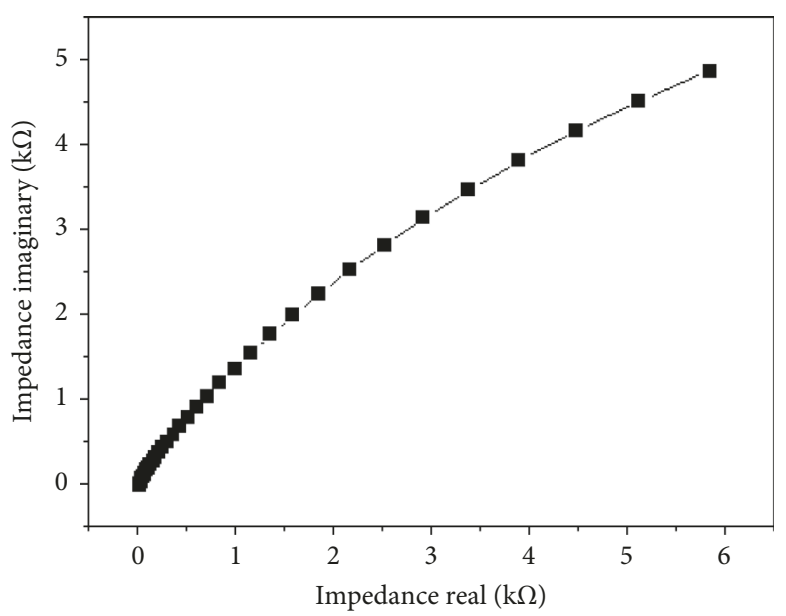

FIGURE 4: Impedance spectrum indicating resistive behavior of HAC-EDTA at higher than 50,000 Hz.

spectroscopy [31], as well as to obtain data related to the conductivity, dielectric coefficient, static properties of the interface, and its dynamic change due to the adsorption and charge transfer phenomena [32].

Furthermore, this technique may help characterize HAC-EDTA as an electroactive polymer [32], since the impedance of these materials can be used for conductivity studies, or even help understand the electrochemical reactions that occur in this hydrogel, aiding in directing this polymer as a biomaterial [31,33].

\subsection{Fourier-Transform Infrared (FTIR) Spectroscopy.}

FTIR is one of the most important tests to study the interaction between calcium, magnesium, and HAC-EDTA since it is possible to identify and identify if there is a formation of characteristic bands after interaction of these elements in direct contact with the hydrogel.

In Figure 5, it is possible to observe the region with a difference in bands, a disappearance observed at $1626 \mathrm{~cm}^{-1}$, and a decrease in bands at approximately $1400 \mathrm{~cm}^{-1}$.

The characteristic free $\mathrm{NH}$ band can be observed in secondary amines, where the bands of $3,350-3,300 \mathrm{~cm}^{-1}$ 


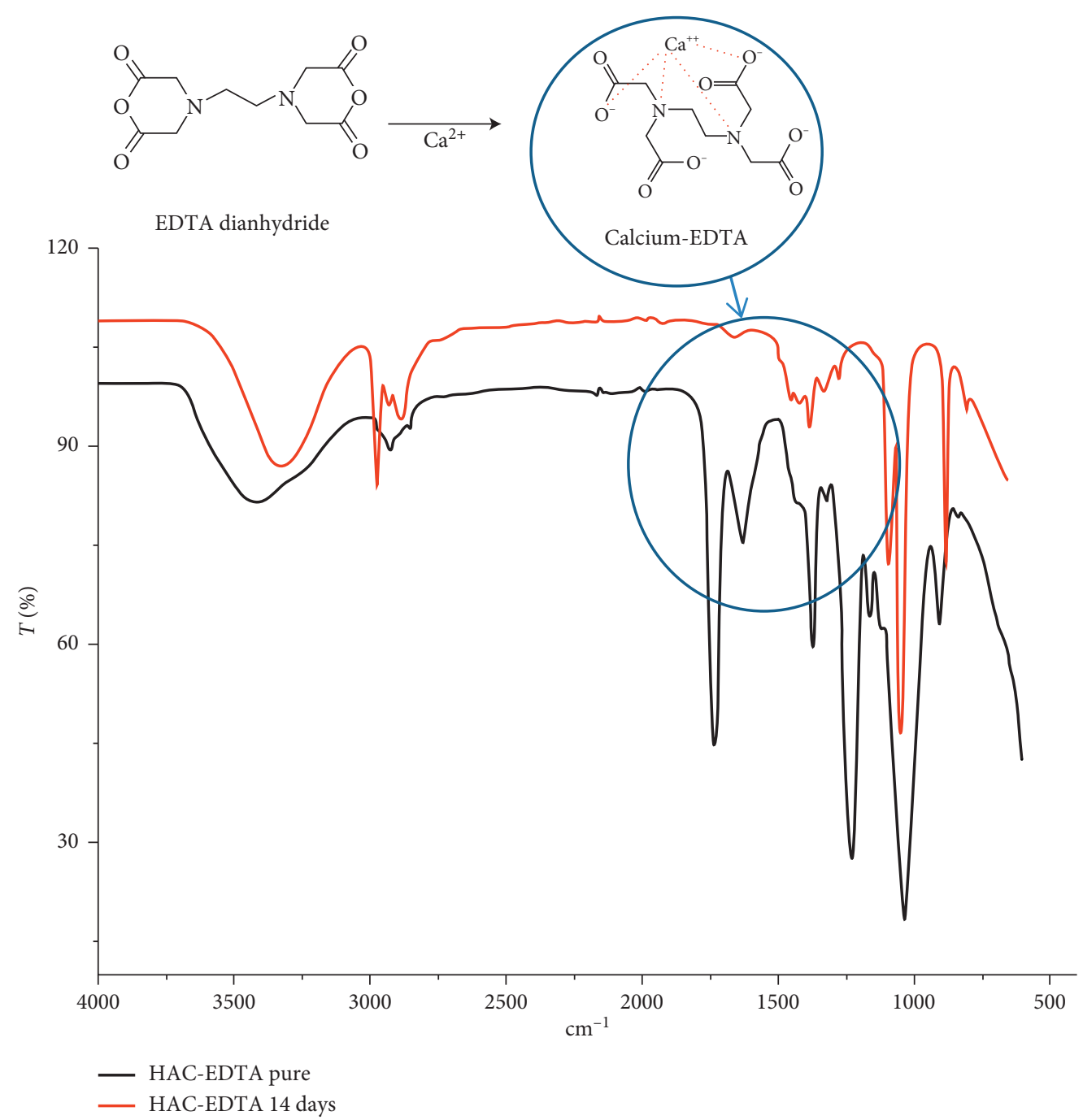

Figure 5: Schematic of interaction of calcium with HAC-EDTA, showing a disappearance of $1626 \mathrm{~cm}^{-1}$ band at approximately $1400 \mathrm{~cm}^{-1}$.

represent aliphatic primary amines, in addition to the reduction of one or two additional bands occurring between 1,650 and $1,600 \mathrm{~cm}^{-1}$ in the presence of conjugated alkenes and finally in the presence of the CX band corresponding to carbon-halogen, which represents a peak only in hydrogel spectroscopy with DMEM after 14 days of direct contact.

Chemistry is fundamental for conducting experiments involving materials; in the case of this work, polymeric materials and biological structures when in the feasibility and cytotoxicity assays express certain responses to the stimuli of contact with the materials.

This interest in the chemistry of organometallic compounds attracted attention when it was observed that the chelating effect of the materials could somehow cause an interaction with the cell culture, and for that reason, it was researched using the FTIR technique if there was notable formation of cyclometrides, for their wide and diversified applications in catalytic processes, and mesogenic materials, in the medical field [34] and more recently in supramolecular chemistry.

According to Trofimenko [35], the formation of cyclometallates describes reactions in which organic binders undergo an intramolecular metallation reaction, which enables the formation of a chelate ring.

Therefore, the formation of a cyclometallate can be attributed to the interaction of HAC-EDTA with $\mathrm{Ca}^{2+}$ and $\mathrm{Mg}^{2+}$, which would justify the fact that, in the first 24 hours of cell culture, the cellular adhesion of mesenchymal cells is impaired and that only after 3 days of cell culture, increased cell adhesion was observed in the material. However, after 7 and 14 days of experiment, it is possible to observe an increase in number of cells adhered to the hydrogel when compared to the negative and positive control. Such results can be corroborated with confocal laser microscopy.

3.3. Dynamic-Mechanical Analysis. The mechanical properties of HAC-EDTA (Figure 6) were determined by mechanical tests for the determination of the glass transition temperature, $T_{\mathrm{g}}$, using the DMA with temperature ramp by the tensile test. [36].

The stresses are calculated from the ratio of the load or force to the cross-sectional area of the specimen. A parameter that quantifies the mechanical strength of a polymer 


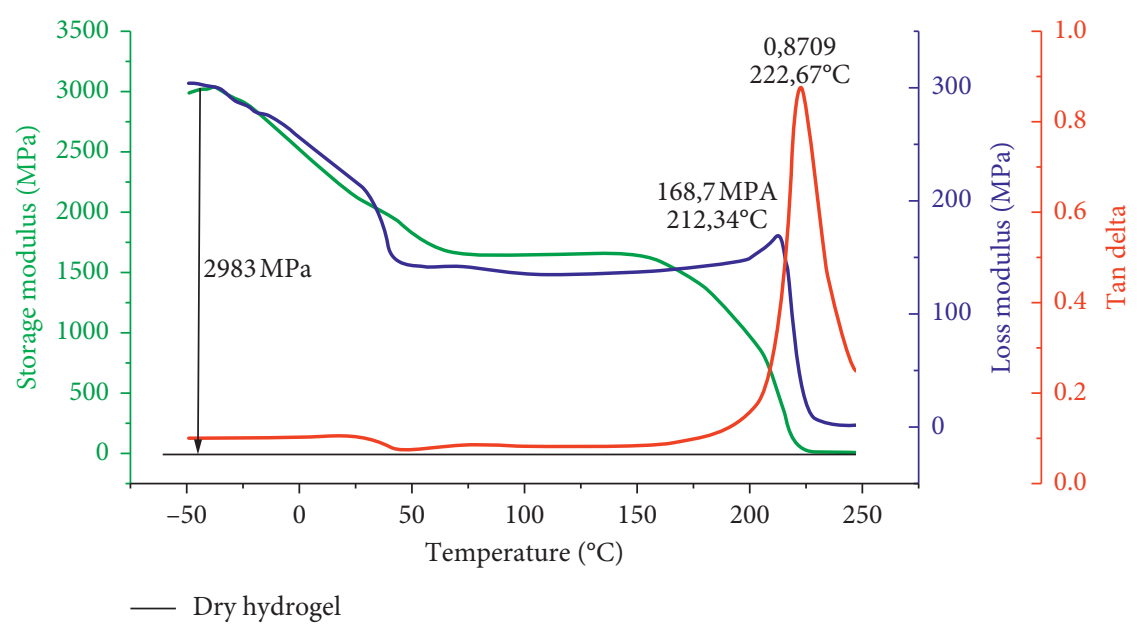

FIgure 6: Dynamic-mechanical analysis curves with a temperature ramp between -50 and $250^{\circ} \mathrm{C}$ for the dry HAC-EDTA film sample.

is Young's modulus, and its definition is related to the rigidity of the material, where the stiffness is directly proportional to the value of the modulus [36].

The dynamic-mechanical analysis was performed by the temperature ramp method. This method of thermal scanning can be performed in single or multiple frequencies [37], and it is the most common method for thermosets to obtain module and $T_{\mathrm{g}}$. In this case, the analysis was performed with a fixed frequency of $1 \mathrm{~Hz}$ for the determination of $T_{\mathrm{g}}$ [37].

In order to verify the influence of water on the secondary transitions indicated in tan delta and $T_{\mathrm{g}}$ of the cellulose acetate, analyses were performed on the dry hydrogel (Figure 6).

As expected, the dry hydrogel sample showed only a delta peak at $222.67^{\circ} \mathrm{C}$, thus confirming the influence of water on the sample.

It can be observed that the frequency effects are more pronounced in the tan delta peaks and that the magnitude of the storage modulus in the vitreous region is quite independent of the frequency so that the frequency effects are more pronounced in the rubbery region [38]. These effects can be observed in Figure 7.

It is possible to observe the disappearance of the first two peaks in the dry hydrogel and a displacement of $T_{\mathrm{g}}$ of the hydrogel in relation to the cellulose acetate. With the crosslinking, the cellulose acetate chains will present a restriction in the movement, and with this greater energy for glass transition, the temperature of $T_{\mathrm{g}}$ will increase.

The characterization of biomaterials goes far beyond biocompatibility, bioinductivity, and controlled drug release dynamics, and it is also important to know the mechanical properties. Hydrogels may have complex viscoelastic characteristics, and therefore, DMA assays were required to evaluate the mechanical behavior of these materials[39].

For polymeric materials whose function will be in the field of biomaterials, negative glass transition temperatures make their application even more interesting since it is known that the ideal human body temperature is $37^{\circ} \mathrm{C}$, which would make it possible to use the biomaterial in
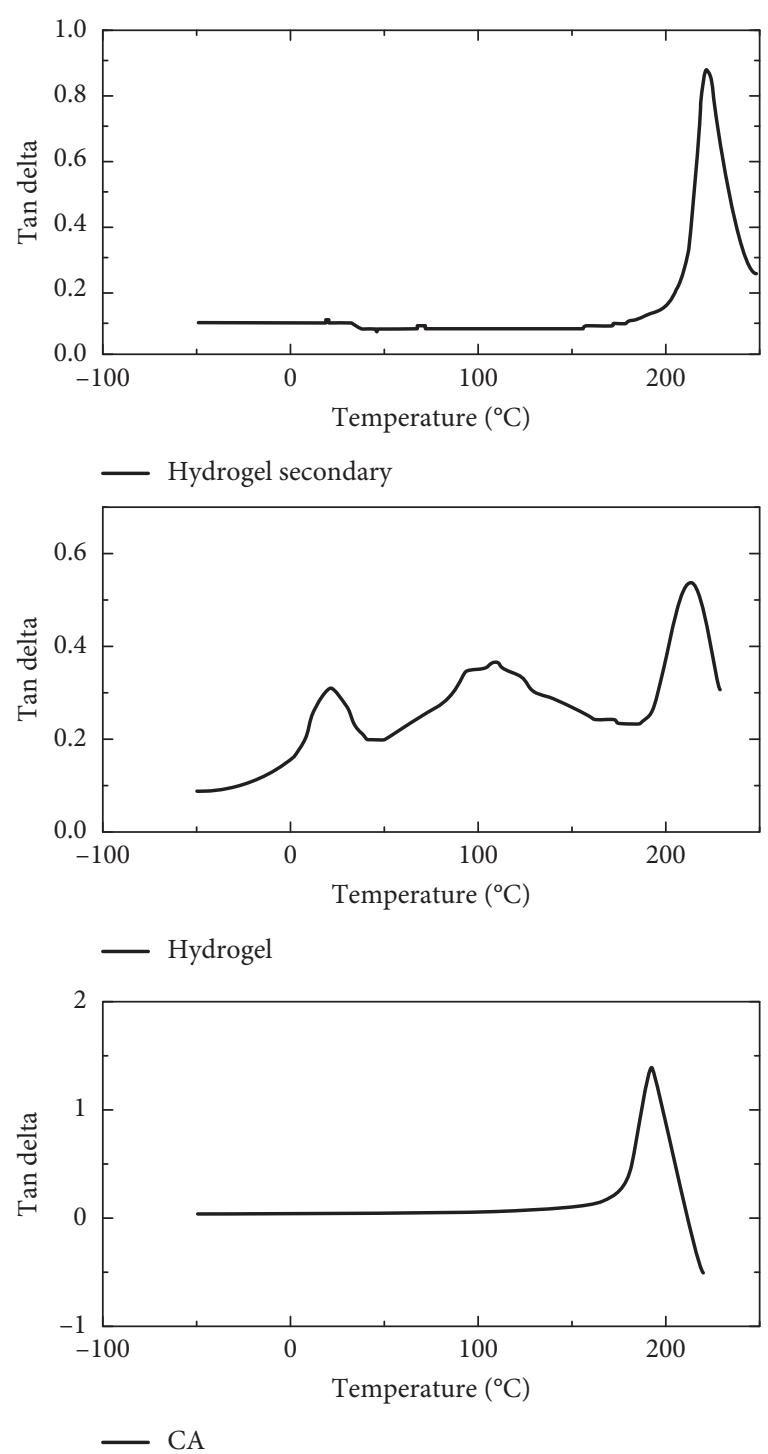

Figure 7: Tan delta dry hydrogel, swollen hydrogel, and cellulose acetate (CA) between -50 and $250^{\circ} \mathrm{C}$. 


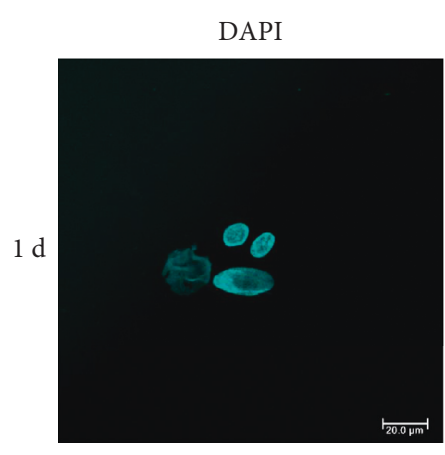

DAPI

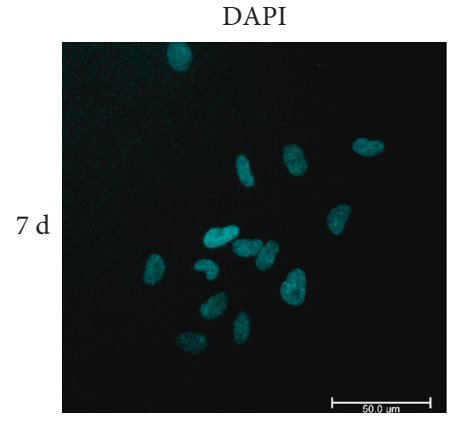

DAPI

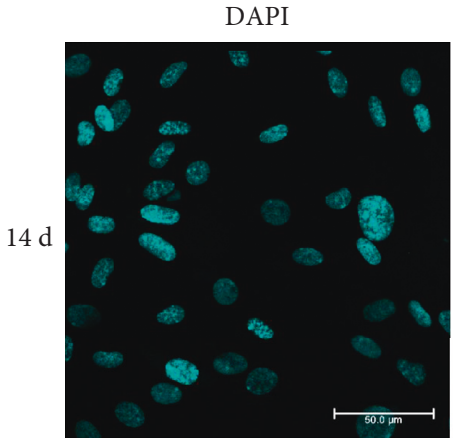

Alexa Fluor 647

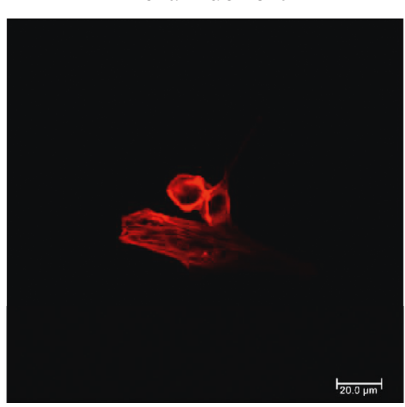

(a)

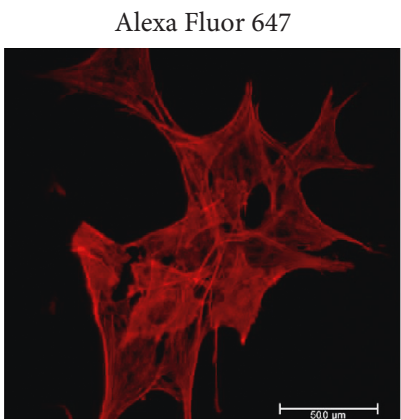

(b)

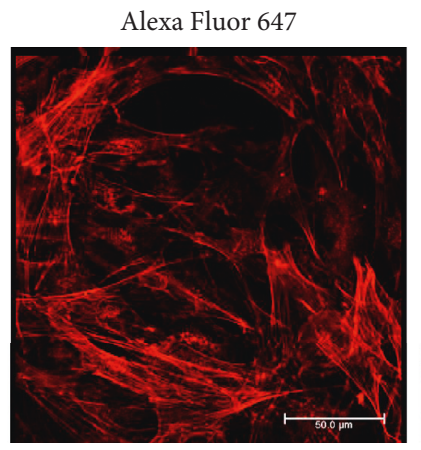

(c)

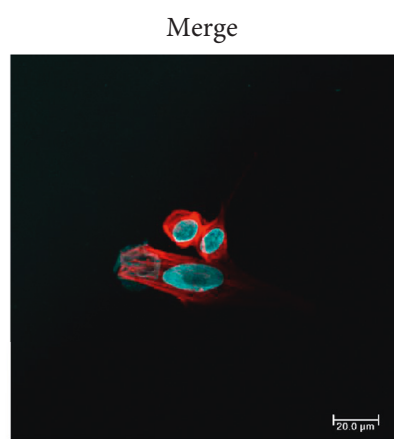

Merge
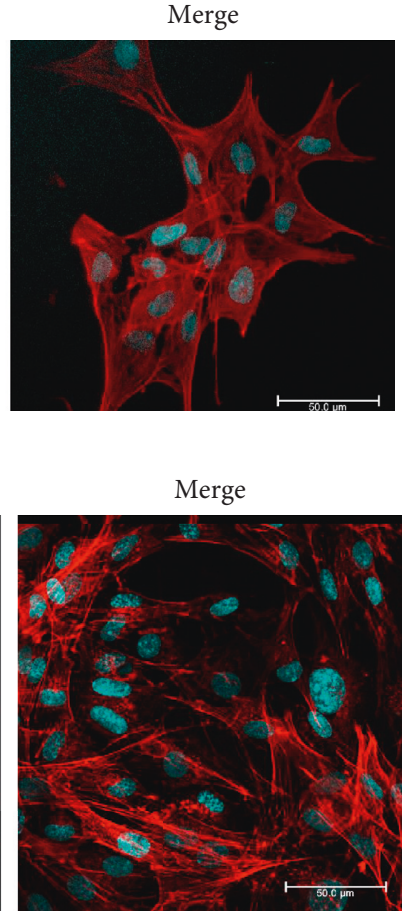

Figure 8: Confocal microscopy analysis of ASC cells adhered to HAC-EDTA at culture times of 1, 7, and 14 days. (a) Cells with low concentration of $\mathrm{Ca}^{2+}$ and $\mathrm{Mg}^{2+}$ on the first day of culture, which represents difficulty in adhesion of cells to the surface of the material. (b) Adhesion was higher when $\mathrm{Ca}^{2+}$ and $\mathrm{Mg}^{2+}$ were added to the material, presenting a larger number of cells on 7 th day of culture. (c) After 14 days, the cells increased in quantity and continued to have morphology of the cell type.

internal or external to the human body without any kind of modification in its structure.

3.4. Confocal Laser Microscopy. The cell viability assay using the HAC-EDTA films as cell growth platform and culture medium with $\mathrm{Ca}^{2+}$ and $\mathrm{Mg}^{2+}$ showed that, in the first 24 hours, the mesenchymal cells presented difficulty of adhesion, which is due to the smaller number of cells adhered to the surface of the hydrogel, and this directly interferes with cellular activity since HAC-EDTA removes $\mathrm{Ca}^{2+}$ and $\mathrm{Mg}^{2+}$, hindering cell adhesion during the first hours of direct contact between the hydrogel and the culture medium and, consequently, between the hydrogel and $\mathrm{Ca}^{2+}$ and $\mathrm{Mg}^{2+}$. However, in the latter results, it can be observed that there is an increase in the amount of cells on the surface of the material, as well as a corresponding morphology with cell adhesion, which presents a scattered appearance.

Although from the beginning of the experiment the DMEM culture medium was changed every 48 hours, there was no decay of the cellular activity before 7 days of culture time and after that time an increase in the cellular activity as well as in the quantity of cells adhered to the surface of the material at 14 days time (Figure 8 ), which justifies controlled release of the hydrogel over time, removing $\mathrm{Ca}^{2+}$ and $\mathrm{Mg}^{2+}$ from the medium and releasing again.

However, the medium exchange justifies a possibility of the hydrogel presenting higher values of $\mathrm{Mg}^{2+}$ (and $\mathrm{Ca}^{2+}$ ) concentration when compared to the DMEM concentration of the positive control, corroborating with the ICP result, published in the previous article of the same research group [18]. 


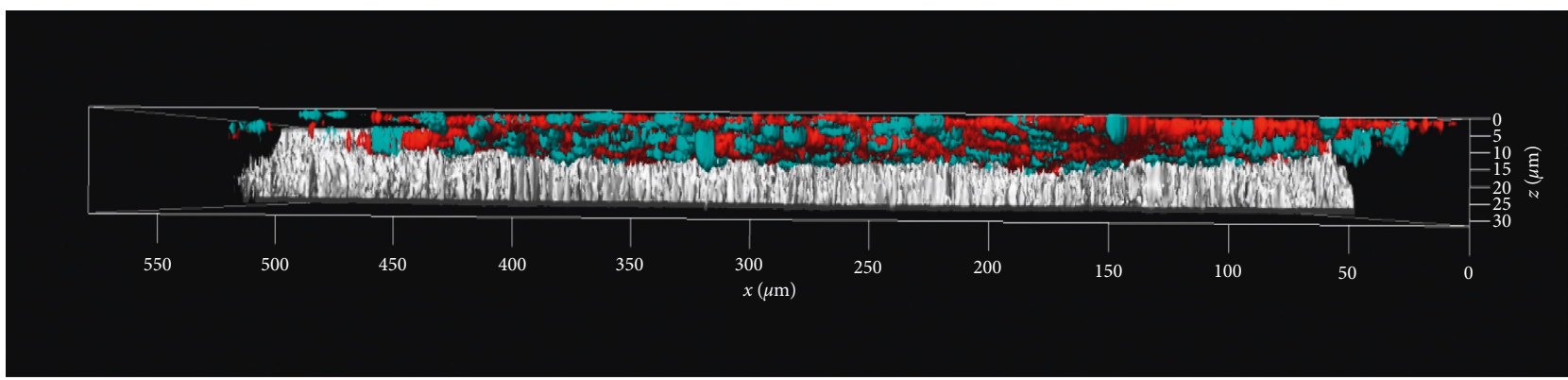

FIGURE 9: 3D reconstruction of HAC-EDTA by confocal laser scanning microscopy. After 14 days, the cell monolayer (blue/red colored) was homogenously adhered over the HAC-EDTA surface (white).

As a result, the exchange of the culture medium every two days, where a new complete culture medium is replaced by the culture medium that has already interacted with the hydrogel and the cells, assists in increasing the concentration of $\mathrm{Ca}^{2+}$ and $\mathrm{Mg}^{2+}$ over time. Thus, one possibility of avoiding cell stress during the first 24 hours of cell culture is saturating the hydrogel films prior to cell viability assays.

Confocal microscopy was also used to characterize the morphology of HAC-EDTA when used as a cellular platform, presenting the adhered cells on its surface.

Materials used as a scaffold in tissue engineering must be well characterized, and confocal laser microscopy is used in the analysis of cellular response by adhesion studies [40].

Cell wall hydrogel arrays of bacterial origin were produced by Favi et al. [41] as tools for the treatment of connective tissue diseases, such as osteoarthritis. For this study, mesenchymal stem cells that adhered to the scaffolds, differentiated, and proliferated were used, demonstrating that the material is cytocompatible [42].

The presence of pores on the surface of HAC-EDTA was not observed, confirming the results of SEM in previous studies [18]. The question was whether this material would be adequate for cell growth; thus, with the help of confocal microscopy, it can be demonstrated that HAC-EDTA presents a suitable surface for cell adhesion (Figure 9) and can be used as a controlled release device, besides its biocompatibility.

The hypotheses derived from the presented results corroborate each other, where EDTA present in the structure of HAC-EDTA is able to form cyclometallates due to its chelating effect using $\mathrm{Ca}^{2+}$ and $\mathrm{Mg}^{2+}$ and then to perform controlled release due to exchange of the culture medium every 48 hours.

Although the best type of scaffold is the extracellular matrix because it presents biological signals and the native environment that cells need for tissue repair [40], the development of biomaterials that can provide these characteristics to cells is still a challenge.

However, in the present work, it is possible to observe that cell adhesion proved by confocal microscopy makes HAC-EDTA a suitable platform for cell growth [42], although in the first days a certain difficulty of adhesion was noticed, a situation that was justified after the tests were performed.

\section{Conclusion}

During the development of this work, little was known about the dynamics of interaction between materials and cells. However, tests have shown that the material has potential to be used as a platform for cell growth and that despite the interaction of HAC-EDTA present in the hydrogel with the salts that make up the culture medium to impair cell adhesion in the first 24 hours, it cannot rule out its use since after saturation, the hydrogel has higher cell adhesion values than the negative control, which is not very common in materials cytotoxicity assays. Much still needs to be studied, especially in the sense of proving the formation of cyclometallates during the experiment. New experiments will be carried out to answer such questions that have arisen in the development of this study.

\section{Data Availability}

All data supporting the findings of this study are available within the article.

\section{Conflicts of Interest}

The authors declare that there are no conflicts of interest regarding the publication of this paper.

\section{Acknowledgments}

This study was financially supported by the Fapesp-São Paulo Research Foundation (2016/19896-2), Federal University of Ouro Preto, and Biomaterials Laboratory, PUCSP, Brazil. We are also grateful to the PPGBMA of UFSCar Sorocaba for conducting the confocal laser microscopy tests and also to the GPML Research Group for DMA analysis.

\section{References}

[1] G. Binyamin, B. M. Shafi, and C. M. Mery, "Biomaterials: a primer for surgeons," Seminars in Pediatric Surgery, vol. 15, no. 4, pp. 276-283, 2006.

[2] B. D. Ratner, A. S. Hoffman, F. Schoen, and J. E. Lemons, "Introduction-biomaterials science: an evolving, multidisciplinary endeavor," in Biomaterials Science: An Introduction to Material in Medicine, B. D. Ratner, A. S. Hoffman, 
J. F. Schoen, and J. E. Lemons, Eds., Elsevier, Academic Press, Cambridge, MA, USA, 3rd edition, 2013.

[3] D. A. Sánchez-Téllez, L. Téllez-Jurado, and L. M. RodríguezLorenzo, "Hydrogels for cartilage regeneration, from polysaccharides to hybrids," Polymers, vol. 9, no. 12, p. 671, 2017.

[4] C. Karakasyan, J. Mathos, S. Lack, J. Davy, M. Marquis, and D. Renard, "Microfluidics-assisted generation of stimuli-responsive hydrogels based on alginates incorporated with thermo-responsive and amphiphilic polymers as novel biomaterials," Colloids and Surfaces B: Biointerfaces, vol. 135, pp. 619-629, 2015.

[5] R. Gelli, M. Scudero, L. Gigli et al., "Effect of $\mathrm{pH}$ and $\mathrm{Mg}^{2+}$ on amorphous magnesium-calcium phosphate (AMCP) stability," Journal of Colloid and Interface Science, vol. 531, no. 1, pp. 681-692, 2018.

[6] A. Yasmeen, R. Ringe, R. Derking et al., "Differential binding of neutralizing and non-neutralizing antibodies to native-like soluble HIV-1 Env trimers, uncleaved Env proteins, and monomeric subunits," Retrovirology, vol. 11, no. 1, p. 41, 2014.

[7] H. Tan and K. G. Marra, "Injectable, biodegradable hydrogels for tissue engineering applications," Materials, vol. 3, no. 3, pp. 1746-1767, 2010.

[8] C. Wang, R. R. Varshney, and D.-A. Wang, "Therapeutic cell delivery and fate control in hydrogels and hydrogel hybrids," Advanced Drug Delivery Reviews, vol. 62, no. 7-8, pp. 699-710, 2010.

[9] A. Chilkoti, M. R. Dreher, D. E. Meyer, and D. Raucher, "Targeted drug delivery by thermally responsive polymers," Advanced Drug Delivery Reviews, vol. 54, no. 5, pp. 613-630, 2002.

[10] V. Giannoulatou, G. S. Theodorou, T. Zorba et al., "Magnesium calcium silicate bioactive glass doped with copper ions; synthesis and in-vitro bioactivity characterization," Journal of Non-crystalline Solids, vol. 500, pp. 98-109, 2018.

[11] A. M. Senna and V. R. Botaro, "Biodegradable hydrogel derived from cellulose acetate and EDTA as a reduction substrate of leaching NPK compound fertilizer and water retention in soil," Journal of Controlled Release, vol. 260, pp. 194-201, 2017.

[12] A. M. Senna, K. M. Novack, and V. R. Botaro, "Synthesis and characterization of hydrogels from cellulose acetate by esterification crosslinking with EDTA dianhydride," Carbohydrate Polymers, vol. 114, pp. 260-268, 2014.

[13] M. Mahmoud, M. Attia, and H. Al-Hashim, "EDTA chelating agent/seawater solution as enhanced oil recovery fluid for sandstone reservoirs," Journal of Petroleum Science and Engineering, vol. 152, pp. 275-283, 2017.

[14] A. A. Mahmoud and H. Al-Hashim, "Insight into the mechanism for oil recovery using EDTA chelating agent solutions from clayey sandstone rocks," Journal of Petroleum Science and Engineering, vol. 161, pp. 625-635, 2018.

[15] Y.-H. Chan, W.-Z. Lew, E. Lu et al., "An evaluation of the biocompatibility and osseointegration of novel glass fiber reinforced composite implants: in vitro and in vivo studies," Dental Materials, vol. 34, no. 3, pp. 470-485, 2018.

[16] N. Shankhwar, M. Kumar, B. B. Mandal, P. S. Robi, and A. Srinivasan, "Electrospun polyvinyl alcohol-polyvinyl pyrrolidone nanofibrous membranes for interactive wound dressing application," Journal of Biomaterials Science, Polymer Edition, vol. 27, no. 3, pp. 247-262, 2016.

[17] D. Atila, D. Keskin, and A. Tezcaner, "Crosslinked pullulan/ cellulose acetate fibrous scaffolds for bone tissue engineering," Materials Science and Engineering: C, vol. 69, pp. 1103-1115, 2016.
[18] A. M. G. Melero, A. M. Senna, J. A. Domingues et al., "Hydrogel based on cellulose acetate used as scaffold for cell growth," International Journal of Chemical and Molecular Engineering, vol. 12, no. 7, pp. 352-362, 2018.

[19] M. Menini, P. Pesce, F. Pera et al., "Biological and mechanical characterization of carbon fiber frameworks for dental implant applications," Materials Science and Engineering: C, vol. 70, pp. 646-655, 2017.

[20] V. Martin and A. Bettencourt, "Bone regeneration: biomaterials as local delivery systems with improved osteoinductive properties," Materials Science and Engineering: C, vol. 82, no. 1, pp. 363-371, 2018.

[21] D. Mantione, I. Del Agua, A. Sanchez-Sanchez, and D. Mecerreyes, "Poly(3,4-ethylenedioxythiophene) (PEDOT) derivatives: innovative conductive polymers for bioelectronics," Polymers, vol. 9, no. 12, p. 354, 2017.

[22] V. H. Fragal, D. M. Catori, E. H. Fragal et al., "Two-dimensional thermoresponsive sub-microporous substrate for accelerated cell tissue growth and facile detachment," Journal of Colloid and Interface Science, vol. 547, pp. 78-86, 2019.

[23] S. Li, X. Pu, X. Chen, X. Liao, Z. Huang, and G. Yin, “A novel bi-phase Sr-doped magnesium phosphate/calcium silicate composite scaffold and its osteogenesis promoting effect," Ceramics International, vol. 44, no. 14, pp. 16237-16245, 2018.

[24] P. K. Singh, V. K. Saharan, and S. George, "Studies on performance characteristics of calcium and magnesium amended alumina for defluoridation of drinking water," Journal of Environmental Chemical Engineering, vol. 6, no. 1, pp. 1364-1377, 2018.

[25] N. Ataollahi, E. Cappelletto, K. Vezzù et al., "Properties of anion exchange membrane based on polyamine: effect of functionalized silica particles prepared by sol-gel method," Solid State Ionics, vol. 322, pp. 85-92, 2018.

[26] S. Feske, "Calcium signalling in lymphocyte activation and disease," Nature Reviews Immunology, vol. 7, no. 9, pp. 690-702, 2007.

[27] M. J. Laires and C. Monteiro, "Exercise, magnesium and immune function," Magnesium Research, vol. 21, no. 2, pp. 92-96, 2008.

[28] Y. Guo, Y. Yu, L. Han et al., "Biocompatibility and osteogenic activity of guided bone regeneration membrane based on chitosan-coated magnesium alloy," Materials Science \& Engineering C, vol. 100, pp. 226-235, 2019.

[29] D. Attili, S. D. McClintock, A. H. Rizvi et al., "Calcium-induced differentiation in normal human colonoid cultures: cell-cell/cell-matrix adhesion, barrier formation and tissue integrity," PLoS One, vol. 14, no. 4, Article ID 0215122, 2019.

[30] M. W. Patchan, J. J. Chae, J. D. Lee et al., "Evaluation of the biocompatibility of regenerated cellulose hydrogels with high strength and transparency for ocular applications," Journal of Biomaterials Applications, vol. 30, no. 7, pp. 1049-1059, 2016.

[31] S. Jafari, R. K. S. Raman, and S. C. Nu, "In-vitro biodegradation and corrosion-assisted cracking of a coated magnesium alloy in modified-simulated body fluid," Materials Science \& Engineering C, vol. 78, pp. 278-287, 2017.

[32] V. Pillay, T. S. Tsai, Y. E. Choonara et al., "A review of integrating electroactive polymers as responsive systems for specialized drug delivery applications," Journal of Biomedical Materials Research Part A, vol. 102, no. 6, pp. 2039-2054, 2014.

[33] C. Kleber, M. Bruns, K. Lienkamp, J. Rühe, and M. Asplund, "An interpenetrating, microstructurable and covalently attached conducting polymer hydrogel for neural interfaces," Acta Biomaterialia, vol. 58, pp. 365-375, 2017. 
[34] J. S. Almeida, I. D. Cerdena, and F. Kneer, "Simultaneous visible and infrared spectropolarimetry of a solar internetwork region," The Astrophysical Journal, vol. 597, no. 2, pp. L177-L180, 2003.

[35] S. Trofimenko, "Cyclopalladation reaction," Inorganic Chemistry, vol. 12, no. 6, pp. 1215-1221, 1973.

[36] S. V. Canevarolo Jr., Polymer Science, Artliber, São Paulo, Brazil, 2002.

[37] N. P. Lorandi, M. O. H. Cioffi, and O. Mohch Jr., "Dynamic mechanical analysis (DMA) of polymeric composite materials," Scientiacum Industria, vol. 4, no. 1, pp. 48-60, 2016.

[38] R. R. M. de Freitas and V. R. Botaro, "Biodegradation behavior of cellulose acetate with DS 2.5 in simulated soil," International Journal of Chemical and Molecular Engineering, vol. 12, no. 7, pp. 347-351, 2018.

[39] R. Kocen, M. Gasik, A. Gantar et al., "Viscoelastic behaviour of hydrogel-based composites for tissue engineering under mechanical load," Biomedical Materials, vol. 12, no. 2, article 025004, 2017.

[40] M. Osorio, I. Ortiz, P. Gañán et al., "Novel surface modification of three-dimensional bacterial nanocellulose with cellderived adhesion proteins for soft tissue engineering," $M a$ terials Science \& Engineering C, vol. 100, pp. 697-705, 2019.

[41] P. M. Favi, R. S. Benson, N. R. Neilsen et al., "Cell proliferation, viability, and in vitro differentiation of equine mesenchymal stem cells seeded on bacterial cellulose hydrogel scaffolds," Materials Science and Engineering: C, vol. 33, no. 4, pp. 1935-1944, 2013.

[42] T. N. Pansani, F. G. Basso, I. D. R. Souza et al., "Characterization of titanium surface coated with epidermal growth factor and its effect on human gingival fibroblasts," Archives of Oral Biology, vol. 102, pp. 48-54, 2019. 


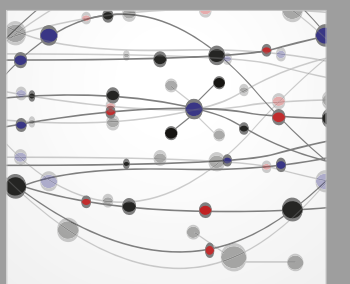

The Scientific World Journal
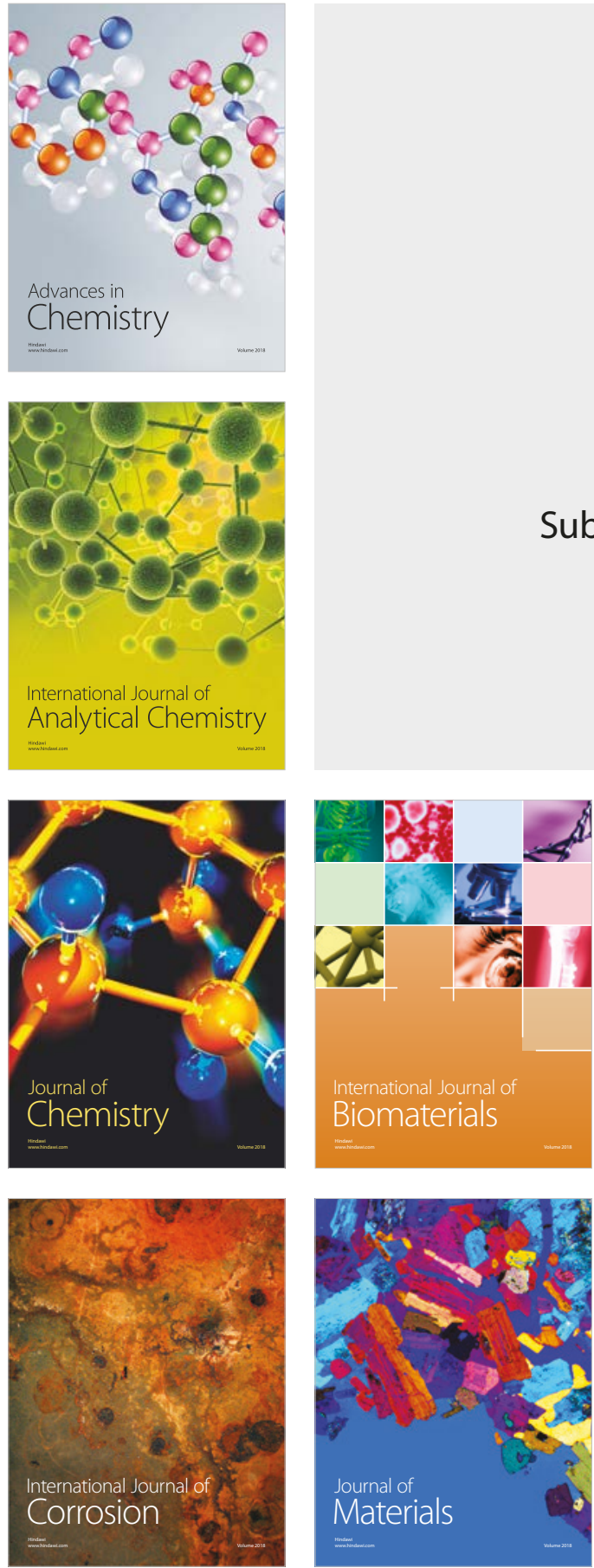

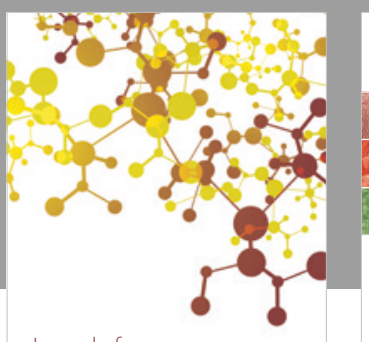

Journal of

Applied Chemistry
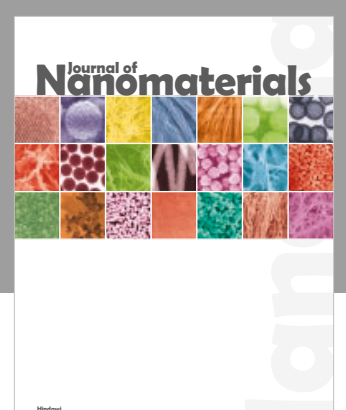

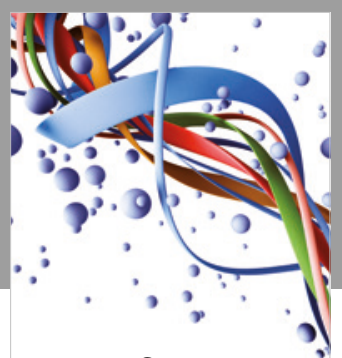

Scientifica

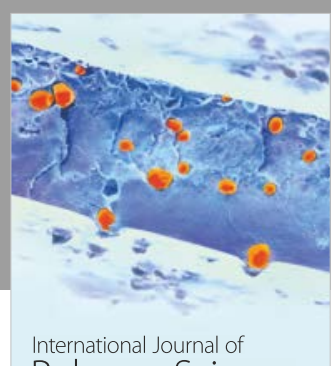

Polymer Science

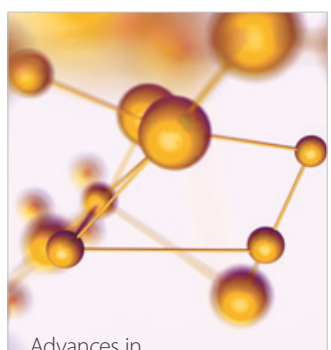

Physical Chemistry
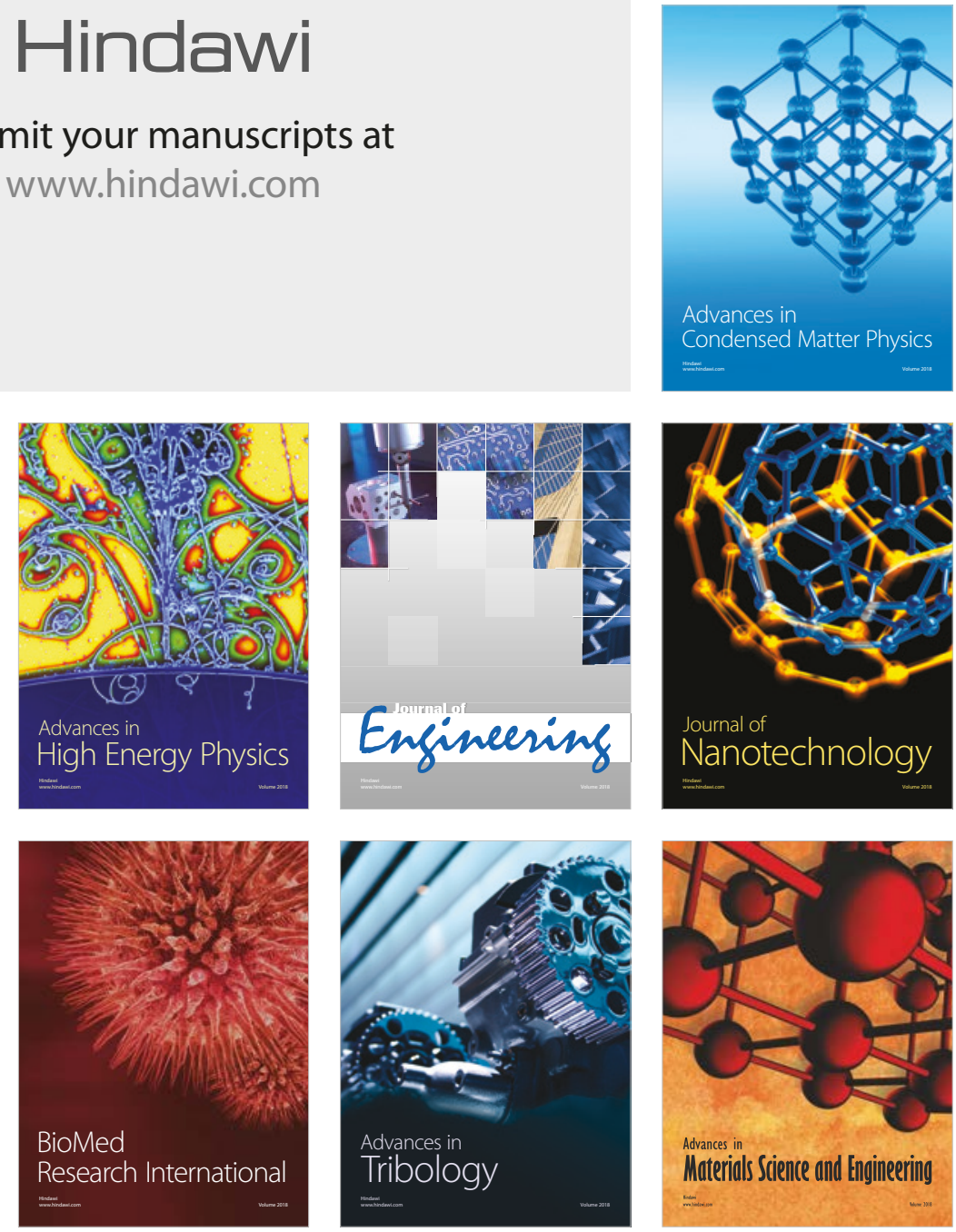